\title{
Publicité, marketing et parfums. Approche psychosociale d'une
} double illusion

In: Communication et langages. N¹36, 2ème trimestre 2003. pp. 43-57.

\section{Résumé}

En s'intéressant aux liens existant entre publicité, marketing et parfums, les auteursétudient deux illusions principales : celledu marketing censé être au service des

produits achetés pour leur odeur et celledes parfums et odeurs réellement mis au

service du marketing. Ils montrent ainsi comment sémiologie et psychologie sociale peuvent s'articuler pour étudier la communication publicitaire. L'image des parfums est construite à partir des désirs profonds et des motivations psychologiques des utilisateurs. C'est la première illusion : motivations, désirs mais également messages publicitaires n'ont rien à voir avec la fragrance et le parfum lui-même. En revanche, les industriels et le marketing associent parfums et marques et utilisent les odeurs dans les points de ventes. C'est la deuxième illusion qui concerne l'influence subliminale du consommateur par des senteurs, dernière étape de ce que Didier Courbet et Marie-Pierre Fourquet appellent l'ère de la « polysensorialité » du marketing et de la communication sur le lieu de vente.

Citer ce document / Cite this document :

Courbet Didier, Fourquet Marie-Pierre. Publicité, marketing et parfums. Approche psychosociale d'une double illusion. In: Communication et langages. $N^{\circ} 136$, 2ème trimestre 2003. pp. 43-57.

doi : $10.3406 /$ colan.2003.3204

http://www.persee.fr/web/revues/home/prescript/article/colan_0336-1500_2003_num_136_1_3204 


\title{
Publicité, marketing et parfums Approche psychosociale d'une double illusion
}

\author{
Didier Courbet et Marie-Pierre Fourquet
}

\begin{abstract}
En s'intéressant aux liens existant entre publicité ${ }^{1}$, marketing et parfums, les auteurs étudient deux illusions principales : celle du marketing ${ }^{2}$ censé être au service des produits achetés pour leur odeur et celle des parfums et odeurs réellement mis au service du marketing. Ils montrent ainsi comment sémiologie et psychologie sociale peuvent s'articuler pour étudier la communication publicitaire.

L'image des parfums est construite à partir des désirs profonds et des motivations psychologiques des utilisateurs. C'est la
\end{abstract}

première illusion : motivations, désirs mais également messages publicitaires n'ont rien à voir avec la fragrance et le parfum lui-même. En revanche, les industriels et le marketing associent parfums et marques et utilisent les odeurs dans les points de ventes. C'est la deuxième illusion qui concerne l'influence subliminale du consommateur par des senteurs, dernière étape de ce que Didier Courbet et MariePierre Fourquet appellent l'ère de la "polysensorialité " du marketing et de la communication sur le lieu de vente ${ }^{3}$.

\section{LE MARKETING ET LA COMMUNICATION DU PARFUM : QUAND LE DÉSIR ESCAMOTE LA SENTEUR}

Devant la multiplicité des offres et des marques, chaque fabricant se doit, d'une part, de démarquer ses produits de ceux des concurrents et, d'autre part, de les adapter parfaitement aux segments de consommateurs visés. Surprenant parfois ceux qui considèrent le parfum comme une « création artistique », ce secteur utilise, comme tous les autres, les démarches du

1. J.J. BOUTAUD, Sémiotique et communication. Du signe au sens, Paris, L'Harmattan, 1998.

2. Le marketing est le « mécanisme économique et social par lequel individus et groupes satisfont leurs besoins et désirs au moyen de la création et de l'échange de produits et autres entités de valeur pour autrui », P. KOTLER et B. DuBOIS, Marketing Management, Paris, Publi-Union ( $6^{\mathrm{e}}$ édition), 1989.

3. D. CouRBET et M.P. FouRQUET, «Les stratégies subliminales du marketing olfactif. Au corps défendant du consommateur... ", in P. LARDELLIER (éd.), À Fleur de Peau : Corps, odeurs et parfums, Belin, pp. 157-165, 2003. 
marketing pour concevoir et commercialiser parfums et eaux de toilette ${ }^{4}$. La communication et la publicité reflètent et mettent subtilement en scène les désirs des individus. Elles n'attachent presque aucune importance à la senteur elle-même et la relèguent à une fonction subalterne ${ }^{5}$.

\section{Processus de choix de l'acheteur et influence publicitaire}

L'image que les industriels vont donner à un parfum particulier est conçue à partir de quelques questions : qu'est-ce qui motive une personne à utiliser ou acheter un parfum ? Comment s'opère le processus d'achat? Mieux on comprend le processus de choix des consommateurs et ses motivations à l'achat, mieux on pourra les influencer. C'est ainsi que le marketing fait appel, d'une part, à des disciplines théoriques comme la psychologie sociale ${ }^{6}$ et d'autre part, à des méthodologies empiriques pour mener études et enquêtes. Ainsi, on peut distinguer deux principaux processus d'achat d'un parfum.

La plus grande partie du premier processus décisionnel s'effectue sans contact olfactif avec le parfum et donc hors du magasin. C'est le cas lorsque l'individu est avant tout séduit par la publicité. Comment est-il possible que la publicité soit aussi efficace alors que les contenus des messages sont aussi éloignés de la caractéristique intrinsèque même des parfums: leur odeur? L'une des principales raisons de ce phénomène est de nature neuropsychologique : la mémoire parvient très mal à mémoriser l'odeur sous forme brute, c'est-à-dire non associée à un support sémantique. L'odeur est donc mémorisée de manière holistique (en une seule forme) en association avec des propositions linguistiques, soit au sein de réseaux sémantiques (qui

4. Pour simplifier, nous utiliserons pour la suite le seul terme de «parfum » pour désigner à la fois le parfum et les eaux de toilette.

5. Au niveau méthodologique, cette partie est la synthèse d'une étude d'un corpus de 185 annonces publicitaires écrites pour des parfums et eaux de toilette féminins et masculins. Ces annonces sont parues dans la presse magazine française entre 1997 et 2002, plus spécifiquement dans les supports: Elle; Marie-Claire; Biba ; Femme Actuelle; Vogue, Cosmopolitan; Max ; NewLook ; Men's Health ; FHM.

6. M.P. Fourquet et D. Courbet, "Les professionnels du marketing", in J.L. Beauvois et J.M. Monteil (dir.), La Psychologie Sociale, tome 5 : Des compétences pour l'application (pp. 301-316), Presses Universitaires de Grenoble, 2001 ; voir également J. R. ROSSITER et L. PERCY, Advertising and Promotion Management, New York, McGraw-Hill, 1997. 
codent par exemple la provenance ou une évaluation affective), soit dans la mémoire épisodique. Cette dernière est la mémoire des événements de vie de l'individu: le parfum peut être associé à un souvenir, une scène ou un lieu chargés émotionnellement, parfois à une personne de notre entourage. Les images mentales créées par le consommateur avant de choisir une marque particulière jouent donc un rôle important.

II est logique dans ces conditions que l'individu en contact avec un parfum parvienne difficilement à analyser avec justesse ses caractéristiques et ses différentes composantes. II est donc facile pour la publicité de proposer des supports sémantiques ou des images qui n'ont plus rien à voir avec la senteur et qui seront mémorisés avec l'odeur et la marque elle-même. Mais le mécanisme d'action de la publicité est encore plus subtil. En mettant en scène des stimuli fortement désirés par la cible (la séduction, l'érotisme, le luxe, la valorisation sociale...), elle flatte tout d'abord une motivation psychologique préexistante chez l'individu. II s'agit cependant de faire en sorte que le consommateur ne soit pas conscient du caractère artificiel et complètement fabriqué de l'association entre la marque et ces stimuli. II est devenu tellement habituel de voir, par exemple, associer parfums et valorisation sociale qu'on ne s'aperçoit plus de l'absence de lien fonctionnel entre les deux. Si le consommateur prend conscience d'un tel mode d'influence, cela provoque une réaction opposée, appelée par les psychologues " effet de réactance ». Non seulement la publicité peut déclencher l'envie de se rendre dans le point de vente pour essayer le produit, mais elle prédispose de plus le consommateur à évaluer favorablement la senteur. La valeur du produit est ainsi presque entièrement donnée par le message publicitaire.

Lorsque l'individu se rend sur le lieu de vente sans avoir déjà arrêté son choix, un second processus s'opère directement dans le magasin. En ayant la possibilité de sentir la fragrance et de voir le flacon, il va évaluer différentes marques et adopter une attitude à l'égard de chacune d'elles. Sans forcément l'expliciter clairement, il effectue tout d'abord une comparaison à partir de différents critères de choix classés par ordre d'importance (1. odeur plaisante ; 2. prix entre 50 et 70 euros ; 3 . marque qui fait une publicité attirante ; 4 . flacon original qui attire l'attention). 
On peut dès lors formuler des hypothèses sur les processus en jeu. Théorisé à partir des travaux de Fishbein et Ajzen ${ }^{7}$ sur les attitudes, le premier processus utilisable est compensatoire. Toujours sans en avoir une claire conscience, la personne donne une « note générale » à la marque en additionnant mentalement les critères qui, pour elle, sont importants. Plus les avantages sont nombreux et primordiaux pour la personne, plus la note sera élevée. Les critères négatifs (comme un prix trop élevé) font, bien sûr, diminuer la « note générale ". Ainsi un avantage sur un certain critère de choix peut compenser un handicap sur un autre. Cette compensation n'est pas possible si les processus utilisés sont éliminatoires. En effet, en suivant un processus dit conjonctif, le consommateur établit des seuils de performance minimale sur chaque critère de choix. II suffit que le seuil d'un seul de ces critères ne soit pas franchi pour que la marque soit éliminée ${ }^{8}$.

Une fois le produit acquis, la personne va éviter toute source de dissonance cognitive. Moins elle est sûre d'avoir bien choisi, plus elle va éviter les informations susceptibles de remettre en cause son achat (la publicité concurrente, les discussions avec ses proches...) et chercher des informations qui confortent son choix (en s'exposant notamment à des publicités de la marque choisie).

\section{Positionnement et publicité : \\ illusions de senteurs et réalités imaginaires}

Se fondant sur la connaissance de ces processus de choix et sur la psychologie des consommateurs, l'élaboration du positionnement (c'est-à-dire de l'image) d'un parfum passe par deux étapes. Après avoir effectué des recherches sur les motivations profondes des consommateurs, il s'agit tout d'abord de classer par ordre croissant les plaisirs et satisfactions recherchés par chacun des consommateurs. Ensuite l'analyse des positionnements des concurrents permet de savoir avec précision quelles sont les motivations déjà couvertes par les autres marques, de manière à

7. M. FISHBEIN et I. AJZEN, Belief, Attitude, Intention and Behavior: An Introduction of Theory and Research, Addison-Wesley, Reading, Mass., 1975.

8. D. GRETHER, L. WILDE, «An analysis of conjunctive choice : theory and experiments ", Journal of Consumer Research, n 10, p. 373-386, March 1984. 
proposer un positionnement original et bien démarqué (un parfum de «niche »). Si le producteur suit rigoureusement une démarche marketing, le flacon et même la fragrance sont alors conçus et fabriqués en fonction de ces études. Pour le compositeur et le designer, un cahier des charges indique comment ils doivent satisfaire les motivations du segment visé. On trouve ainsi trois grandes familles de positionnement et donc trois grandes familles de contenu publicitaire.

\section{Les positionnements reposant sur des désirs psychologiques}

Le mode d'action des publicités pour cette famille de produits est essentiellement de nature affective. L'objectif est de déclencher des émotions positives pour provoquer une attitude favorable au message, une adhésion du consommateur ainsi qu'une sympathie pour la marque. De multiples procédés publicitaires sont stratégiquement fabriqués dans ce but (musiques, signes chargés symboliquement et émotionnellement, couleurs $\left.{ }^{9} . ..\right)$. Le processus de réception de la publicité et d'influence sur une personne dans un état affectif positif est alors tout à fait particulier. Celle-ci ne juge pas le message en fonction d'une analyse détaillée des arguments ou du contenu, mais de manière beaucoup plus globale, à partir d'un sentiment général. Les émotions positives conduisent ainsi à évaluer rapidement le message avec des règles simples de décision (appelées heuristiques). Les conclusions tirées par le récepteur sont d'autant plus favorables au contenu que celui-ci montre une scène séduisante ou chargée affectivement. Autrement dit, la publicité est d'autant plus efficace qu'elle met en scène un produit judicieusement positionné à partir des plus forts désirs des consommateurs, conscients ou non. À l'intérieur de cette famille, le marketing et la publicité exploitent trois types de désirs et de motivations.

\section{Narcissisme et carte d'identité olfactive}

Les personnes qui utilisent le parfum pour aider à se construire une identité et une image de soi valorisées sont sensibles aux

9. D. Courbet, Puissance de la Télévision, Stratégies de communication et influence des marques, L'Harmattan (Coll. « Communication »), Paris, 1999. 
publicités qui montrent une image séduisante du consommateur typique. Dans les stratégies marketing indifférenciées qui ne visent pas un segment précis de consommateurs, la publicité montre rarement les visages des personnes. Au contraire, dans les stratégies segmentées, la publicité cherche à faire en sorte que les utilisateurs s'identifient à une personne dont le profil est bien marqué et qui incarne des idéaux culturels et sociaux. Selon la conception psychanalytique du narcissisme, c'est l'amour que le sujet se porte à lui-même qui le pousse à se parfumer. Le parfum devient un moyen de satisfaire une quête d'amour de soi-même pour soi-même mais également une quête d'amour des autres pour soi-même. En utilisant cette marque, le consommateur cherche à ressembler au personnage qu'il voit dans le message publicitaire, personnage qu'il désire tant, qui est tant désiré par les autres et auquel le consommateur désirerait tant ressembler.

Grâce aux concepts de soi et d'identité sociale, une approche psychosociale permet de montrer que le parfum peut devenir une « carte d'identité olfactive » et même participer directement à la construction de l'identité de l'individu. En utilisant tel parfum, le consommateur souhaite se donner une certaine image et donc une identité aux yeux des autres mais aussi à ses propres yeux. Le mode de communication que la personne va adopter avec autrui peut reposer sur cette représentation identitaire. Une marque de parfum peut ainsi être intimement liée à sa propre identité, identité que l'on veut évidemment unique. C'est ce qui explique que l'utilisateur d'une marque n'apprécie pas qu'une autre personne utilise le même parfum que lui. Le designer du flacon et le stratège en communication jouent bien sûr de ce "fantasme d'assimilation », par exemple en sculptant le flacon de manière à ce qu'il ressemble à la représentation humaine désirée.

\section{Luxe et valorisation sociale}

La valorisation et la distinction sociales sont une autre motivation d'achat du parfum. Dans les narrations publicitaires, l'univers luxueux dans lequel est consommé le produit flatte essentiellement cette motivation. Par la consommation de produits à prix élevés, l'individu souhaite montrer ou faire croire aux autres qu'il appartient à une catégorie sociale 
supérieure. Cette famille de consommateurs est alors sensible aux symboles du luxe que l'on va donc insérer dans les publicités ou qui peuvent être connotés dans le nom de la marque : voiture haut de gamme, bijoux, vêtements de haute couture... II semblerait logique que les personnes les plus sensibles à ce type de positionnement soient celles qui attachent une grande importance à l'opinion des autres et qui sont préoccupées par l'image qu'elles donnent d'elle-même à autrui ${ }^{10}$. Pour les produits dont le positionnement repose sur le luxe, on sait depuis les travaux de l'économiste Veblen que si le prix du produit baisse, les ventes baissent également : certains consommateurs n'achètent le parfum que si son prix est élevé.

\section{Séduction et désirs sexuels}

Sans entrer dans le débat concernant la puissance et la place de ces désirs dans les comportements humains, il est indéniable qu'une envie de séduction sous-tend l'utilisation du parfum. Le marketing ne s'intéresse pas aux réels effets aphrodisiaques de la senteur mais essentiellement aux représentations que le consommateur se fait du produit. La narration publicitaire peut montrer ouvertement l'acte de séduction, le suggérer ou faire comprendre qu'il va se dérouler. On retrouve ainsi dans de nombreuses publicités le mythe de la personne séductrice que le parfum rend irrésistible.

\section{Les positionnements reposant sur des modes et des valeurs sociales du moment}

Pour contourner la saturation des marchés et la difficulté à se démarquer des concurrents, les fabricants ont récemment lancé un nouveau type de territoires conceptuels : les positionnements reposant sur les modes. La mode étant éphémère, ce type de positionnement ne peut pas durer. Ainsi, les fabricants font en sorte qu'une grosse quantité de produits se vende immédiatement. Le succès s'obtient alors avec une fragrance qui plaît au plus grand nombre. L'intensité publicitaire et le large ciblage imposent un important budget de communication 
(CK One a investi près de 500 millions de francs lors de son lancement). La récente mode de l'individu « androgyne » a par exemple engendré l'apparition de plusieurs eaux de toilette « unisexe ». Les modes de la « transparence » et des régimes diététiques hypocaloriques ont permis également de lancer de nombreux produits positionnés notamment à partir du concept de l'eau (Eau d'Eden de Cacharel en 1996).

\section{Les positionnements reposant sur le parfum « création artistique »}

Plusieurs études marketing ont montré que certains segments de consommateurs recherchent un parfum qui soit une véritable création artistique et une œuvre d'art. Refusant d'acheter des " parfums de masse » ou des «produits marketing ", ils cherchent une fragrance audacieuse, au caractère marqué, conçue à partir d'ingrédients naturels. Offrant alors de réelles innovations olfactives, les marques proposent donc à ces consommateurs de porter un parfum « confidentiel », né non pas des tests ou des études marketing, mais d'un long processus de création artistique... ce qui devient alors son positionnement marketing et son argument publicitaire (Thé pour un été de L'Artisan Parfumeur). Comme la principale motivation latente est ici la distinction sociale, concrétisée par la rareté du produit, la publicité doit être finement ciblée, discrète et en aucun cas massive.

Ainsi dans cette première illusion, l'image du parfum et sa publicité n'ont plus rien à voir avec la fragrance : au cœur même du produit et de la publicité se trouvent, tel un miroir, les désirs des consommateurs. Lorsque le psychologue s'intéresse au secteur de la publicité et du parfum, il est également attiré par un deuxième type d'illusion qui, bien que distinct du premier, lui est explicitement complémentaire. Si le marketing a mis au service de la communication des parfums un impressionnant arsenal de stratégies, techniques et tactiques créatives, l'odeur est par ailleurs devenue une nouvelle arme du marketing. La deuxième partie montre, tout d'abord, comment les industriels et les stratèges en marketing associent parfums et marques et utilisent les odeurs dans leurs "stratégies de communication " sur le lieu de vente pour mieux influencer, souvent à leur insu, les consommateurs. Il s'agit de la seconde illusion. 


\section{LES PARFUMS AU SERVICE DU MARKETING : ILLUSION ET TENTATION COMMERCIALES}

Bien que les consommateurs n'en aient pas explicitement conscience, les stratèges en marketing parfument les produits et les lieux de vente pour mieux influencer leurs achats. Ils créent bien là une illusion puisque les consommateurs se croient libres de leurs choix. Le marketing est bien entré dans une ère «polysensorielle».

\section{Les effets des parfums sur les évaluations et les comportements des consommateurs}

En psychologie environnementale, on définit une odeur à partir de trois dimensions. La première est la qualité affective d'un parfum, son aspect désagréable versus agréable. Une odeur est également définie par sa nature stimulante, c'est-à-dire sa capacité à provoquer des réponses physiologiques chez l'individu. La troisième dimension est l'intensité du parfum. Ces trois dimensions ne sont pas indépendantes. Le caractère stimulant d'un parfum est en effet quasi systématiquement associé à sa dimension affective. Que se passe-t-il dans l'esprit de la personne qui, dans un lieu de vente donné est face à un produit ou dans un espace parfumé artificiellement ${ }^{11}$ ? La psychologie socio-cognitive va nous aider à répondre à cette question.

\section{Une approche socio-cognitive de l'influence des odeurs}

Pour mieux comprendre l'effet d'une odeur sur un individu dans les rayons d'un hypermarché, nous lions les recherches sur l'olfaction et celles sur l'influence et le changement d'attitudes en psychologie sociale ${ }^{12}$. Le client traite les informations dans le point de vente de deux façons. S'il a le temps et s'il est impliqué par son achat, il met en place un traitement systématique et approfondi examinant les arguments et les attributs des différentes marques. Cela lui demande un effort cognitif important : il va par exemple lire les indications sur les différentes étiquettes des produits, comparer les différents produits... L'odeur est alors une information supplémentaire

11. Voir également COURBET et FOURQUET, 2003, op. cit.

12. S. CHAIKEN et Y. TROPE (eds.), Dual-Process Theories in Social Psychology, The Guilford Press, 1999. 
qu'il peut prendre en compte pour se former une représentation du produit. Dans ce cas, il en tient compte de marière rationnelle.

Dans un hypermarché, le plus souvent le consommateur est peu impliqué par les produits et ne veut pas passer trop de temps pour faire ces achats. L'odeur d'un produit parfumé influence les acheteurs en leur faisant prendre leur décision beaucoup plus rapidement. En effet, lorsqu'il est pressé ou peu impliqué, le consommateur met en route des traitements demandant peu d'efforts cognitifs : les traitements heuristiques. Ceux-ci ne tiennent pas compte des arguments rationnels liés au produit. Les odeurs influencent les individus en incitant à raccourcir, à leur insu, leur processus de décision et à utiliser des heuristiques, c'est-à-dire de règles de décisions simples (et non forcément fiables) : "cette lessive sent le frais : elle doit donc être efficace ».

Les études expérimentales sur les effets des odeurs montrent que l'illusion fonctionne remarquablement. Même quand les acheteurs n'ont pas véritablement conscience de l'odeur, Spangenberg et al. ${ }^{13}$ indiquent que la présence d'un parfum ambiant non déplaisant dans un point de vente affecte positivement :

- les évaluations du point de vente lui-même : il est mieux évalué, davantage apprécié et son image perçue comme étant plus moderne ;

- les évaluations de l'environnement au sein du point de vente : il est perçu comme étant plus gai, plus plaisant, plus animé ;

- les évaluations des produits vendus.

\section{Les odeurs et l'évaluation affective du consommateur}

La perception et la mémorisation des odeurs sont intimement liées aux émotions. Voilà pourquoi la dimension affective de l'odeur a fait l'objet de plusieurs études en marketing. Les recherches sur les effets des parfums localisés ou ambiants mettent en évidence les actions favorables du parfum notamment sur l'affect des individus. Par exemple, ces effets influencent favorablement

13. E.C. Spangenberg, A.E. Crowley, P.W. Henderson, « Améliorer l'environnement magasin: les signaux olfactifs affectent-ils les évaluations et les comportements?", Recherche et Applications en Marketing, vol. 11, $n^{\circ}$ 4/96, pp. 71-92, 1996. 
la conduite de négociations commerciales ${ }^{14}$ : un vendeur parvient mieux à influencer un acheteur potentiel dans un lieu parfumé. Même si leurs supports empiriques restent largement insatisfaisants, certaines études montrent l'impact favorable des odeurs sur les dépenses monétaires: les consommateurs achèteraient davantage dans un milieu parfumé.

Les impacts bénéfiques sur le stress et sur l'impression de relaxation sont aujourd'hui bien démontrés. Probablement inspirées des partisans de l'aromachologie ou même de l'aromathérapie, des lignes entières de produits de soins et de cosmétiques sont d'ailleurs aujourd'hui positionnées, à partir des vertus attribuées à leurs parfums, sur la relaxation ou l'antistress.

L'odeur suscite des réponses physiologiques avant même de pouvoir être détectée consciemment; on comprend l'intérêt qu'elle présente pour favoriser l'achat d'impulsion tant recherché par les grandes surfaces. Ainsi, le parfum du produit ou de l'environnement d'achat contribue à la création d'un climat favorable à l'achat spontané (comme l'achat de bonbons qui se trouvent à proximité des caisses des hypermarchés). L'effet sur les réponses physiologiques avant toute prise de conscience de la part des individus fait entrer la problématique des effets de l'odeur dans le champ controversé de l'influence subliminale. Dans une recherche de référence, menée par Laird dans les années trente, on a demandé à des consommateurs de juger de la lingerie, certaines pièces étant parfumées et d'autres pas. Les préférences des sujets se sont davantage portées sur la lingerie parfumée sans que ceux-ci aient conscience de l'impact du parfum sur leur choix. Mieux, leur choix, selon eux, serait lié à la bonne texture, à l'éclat ou encore à la bonne solidité perçue de la lingerie parfumée. Ce phénomène que nous qualifierons de « mésattribution " de l'odeur à des caractéristiques propres au produit, n'est pas sans rappeler celui de mésattribution de la familiarité ${ }^{15}$ bien connue en publicité. II ouvre d'intéressantes

14. Voir V., P. Barbet, N. Guichard,, C. Lecoquierre, J.M. Lehu, R. Vanheems, Le marketing olfactif, Paris, Les presses du management, 1999.

15. D. CouRBET, L'influence non consciente de la publicité, dans, D. Courbet et M.P. FoURQUET (dir.) La Télévision et ses influences, De Boeck Université (coll. "Médias Recherche-INA "), à paraître. 
pistes de recherche sur l'influence des odeurs: sans en avoir conscience, l'acheteur attribuerait à des qualités propres au produit les impressions positives qu'il ressent face au produit et qui viennent, en fait, uniquement de l'odeur artificielle.

\section{L'influence du parfum sur le comportement}

Spangenberg et al. ont également testé l'impact positif de la présence de parfum ambiant non déplaisant sur un certain nombre de comportements de consommateurs tels que l'intention d'aller dans le point de vente, le temps passé en magasin (perçu et réel), l'examen des étiquettes de produits ou encore l'intention d'achat de produits. Si le fait que le point de vente soit parfumé ou non n'agit pas sur le temps effectivement passé en magasin, il a un impact sur la durée perçue. Le temps passé est sous-estimé dans l'environnement parfumé. On voit l'application de tels résultats pour les distributeurs qui cherchent sans cesse à rendre agréable une tâche (faire les courses) souvent perçue comme contraignante et désagréable par les clients. Cette distorsion de la perception du temps pourrait par exemple rendre moins pénible l'attente aux caisses.

\section{Le parfum : une nouvelle arme des stratégies marketing}

\section{La politique de produit et les parfums}

Les odeurs de certains produits sont reconstituées ou renforcées afin de souligner une caractéristique essentielle de leur positionnement : ajout d'arômes dans le secteur agroalimentaire ou dans les produits de nettoyage. Ces derniers ont souvent des odeurs symboliquement associées à la propreté telles que le pin ou le citron. Cette exploitation est également fréquente dans des garages par exemple, avec des voitures d'occasion souvent parfumées « au neuf » ou encore dans des magasins de chaussures où on rajoute une odeur de cuir pour rassurer sur la qualité des marchandises vendues. Parfois une odeur, surprenante au regard du produit qu'elle accompagne, peut-être à la base du positionnement ; ainsi, dans une étonnante opération de co-branding, un fabricant de produits de toilette et une célèbre marque de bonbons se sont associés pour lancer une gamme de bains moussants parfumés aux bonbons pour les enfants. D'autre part, certains produits sont 
parfumés dans le but d'attirer l'attention du consommateur. Plusieurs marques ont commercialisé avec succès des gammes de sous-vêtements féminins parfumés. L'exploitation de ces signaux olfactifs pour attirer l'attention des personnes sur un produit particulier semble donc efficace. Si l'odeur améliore l'efficacité perçue d'un produit, elle peut également renforcer les performances « réelles» du produit. Barbet et $a .^{16}$ citent à ce titre le fil de pêche à la vanille pour mieux attraper les carpes qui en raffolent ou encore les sacs poubelles parfumés au citron pour chasser les « mauvaises odeurs » et éloigner les chiens. L'augmentation de la performance du produit est parfois indirecte : pour lutter contre les caries des enfants, il vaut mieux fabriquer des dentifrices parfumés à la fraise. Les enfants les utilisent plus volontiers.

Certaines odeurs peuvent également servir à masquer d'autres odeurs. On utilise par exemple cette technique dans les entreprises dont l'activité engendre des odeurs désagréables qu'il convient de masquer avec des odeurs agréables, tant pour le bien-être des salariés que pour l'image de l'organisation auprès de publics externes. Des produits d'usage fréquent à l'odeur désagréable comme les carburants sont également concernés ; un pétrolier a d'ailleurs parfumé son gasoil avec une odeur... neutre. L'odeur de substitution doit cependant être en accord avec l'image du produit ; un autre pétrolier l'a appris à ses dépens en parfumant son essence à la fraise, association qui est apparue comme incongrue dans l'esprit du consommateur. Le pétrolier l'a immédiatement retirée.

\section{La politique de distribution et les parfums}

En hypermarché, le nombre élevé de références et de rayons exige une utilisation discrète des signaux olfactifs. $\grave{A}$ défaut, on créerait vite un «brouhaha olfactif » indigeste. Ainsi, si une odeur marine peut motiver à l'achat dans le rayon poisson, elle risque d'apparaître comme un frein dans les rayons pâtisserie ou textile voisins. Les hypermarchés exploitent parfaitement, et de façon discrète, les odeurs au cours d'animations saisonnières. En effet, diffuser une odeur artificielle de chocolat stimule les achats lors des opérations de Pâques ou Noël, alors 
que des odeurs de noix de coco et de sable dynamisent les ventes de produits bronzants ou de maillots de bain. Ces diffusions sont d'autant plus efficaces qu'elles se font dans le cadre d'une animation à la fois visuelle (décors de sapins enneigés ou de sable fin plantés de parasols), sonore (chants de Noël ou musique Hawaïenne) gustative et/ou tactile (dégustation de chocolat ou essai des produits bronzants).

Dans les lieux de distribution où il y a un lien commun entre l'ensemble des produits, le parfum ambiant peut être un facteur de différenciation par rapport aux concurrents. Ainsi, au même titre que les codes couleurs, l'aménagement et la signalétique du magasin ou les tenues du personnel, le parfum est une composante de l'identité de l'enseigne et contribue à la création d'une ambiance favorable à l'achat. L'enseigne Nature \& Découvertes a bien saisi l'importance de la polysensorialité. Dans ces points de vente, le prospect a ses cinq sens en éveil. Outre la succession d'univers visuels où, pour la plupart des références, les produits peuvent être touchés, une musique et des odeurs congruentes sont diffusées et des boissons offertes. L'ensemble est systématiquement en rapport avec le positionnement et l'image de l'enseigne.

\section{Communiquer par le parfum}

En matière de publicité, l'exploitation des odeurs est surtout faite dans le média presse écrite. Chacun a déjà senti les publicités magazines pour un parfum, une lessive ou un adoucissant qui proposent des «échantillons olfactifs». Dans ce cas, l'odeur est un argument supplémentaire d'autant plus important qu'elle est un critère discriminant dans le processus de choix du consommateur. Dans les autres médias, les expériences olfactives restent exceptionnelles et ponctuelles (déclenchement de la diffusion d'odeurs via internet ou, moins récemment, la diffusion du film Le Grand Bleu dans des effluves marines lors de son lancement). En communication hors médias, de plus en plus d'entreprises associent leur marque avec une odeur congruente avec leur positionnement. Elles l'utilisent par exemple pour parfumer les lettres commerciales dans le cadre des opérations de publipostage. Ce type de démarche montre une nouvelle fois le rôle que peut jouer l'odeur dans l'image de marque d'une organisation. En effet, au même titre que le 
slogan ou le sonal, le « logolf » ou logo olfactif, permet de traduire l'image d'une marque ou d'une entreprise. Cependant le choix de l'odeur n'est pas simple. Comment choisir une odeur qui soit pertinente avec l'image de l'entreprise ? Air France a tenté l'expérience en se créant une identité olfactive qui soit en harmonie avec son image. Sa signature olfactive est notamment présente dans les pochettes de rafraîchissement données aux passagers des vols ou dans les agences et les comptoirs. La mémoire olfactive étant largement épisodique, on comprend l'intérêt pour une marque d'être associée à une odeur spécifique qui déclenche une émotion positive. De la même façon que les odeurs de craies peuvent réveiller nos souvenirs d'écoliers ou que les parfums de barbe à papa et de praline rappellent les fêtes foraines, sentir la petite pochette Air France dans un autre contexte rappellerait le voyage et permettrait une meilleure mémorisation de la marque. Pour choisir leur signature olfactive, les firmes ayant un rayonnement mondial devront prendre garde aux représentations culturelles des odeurs; les connotations et symboles sont variables selon les cultures. II est donc plus prudent de tester les odeurs, de la même façon que l'on teste la pertinence de noms de marque ou de codes couleurs sur les différentes cibles.

L'exploitation des odeurs à des fins d'influence est un sujet dont les entreprises parlent avec difficulté : elle reste encore confidentielle. De même, les techniques utilisées par les publicitaires et les hommes de marketing pour la conception et la communication des parfums sortent rarement des agences ou des départements marketing. Les psychologues expliquent en effet que lorsque les individus savent comment on les manipule, l'effet de réactance opère et l'influence est inhibée. Toutefois, les deux illusions dont nous avons parlé dans cet article posent des questions d'ordre éthique, renvoyant aux craintes de manipulation des consommateurs par les industriels. En effet, l'influence par les odeurs ou par les désirs profonds des individus, souvent non consciente, suscite des interrogations relatives à la liberté individuelle : jusqu'où peuvent aller ces techniques manipulatrices appliquées à l'insu des individus? 